\title{
Development of a Public Database of Raw Data From a Blood Pressure Cuff
}

\author{
Jiri Jilek \\ Carditech \\ Culver City \\ California \\ USA \\ jilekj@usa.net
}

\author{
Milan Stork \\ Department of Applied Electronics and \\ Telecommunications/RICE \\ University of West Bohemia \\ Plzen, Czech Republic \\ stork@kae.zcu.cz
}

\begin{abstract}
Pressure data acquired from a blood pressure cuff during a blood pressure test are the starting point of noninvasive, single cuff blood pressure determination. Most blood pressure devices on the market use the single cuff method. This paper describes acquisition, analog and digital processing, and storage of raw data from a blood pressure cuff during gradual cuff deflation. Raw data from 30 arm cuff tests and 20 wrist cuff tests were organized to form a database of text files. Separate annotation documents were also created. The database was uploaded to the Google Drive. The database can be downloaded by any interested party. The data can be used for the development of algorithms for blood pressure determination and for the assessment of some cardiovascular variables.
\end{abstract}

Keywords - Blood pressure (BP) cuff, raw data, database, cuff pulse, data files, $B P$ algorithms, cardiovascular variables, Carditech.org

\section{INTRODUCTION}

Blood pressure (BP) measurement is the most commonly performed screening and diagnostic test, not only in the medical practice but also in the home. Single cuff automatic BP monitors are the most prevalent BP devices on the market today. Most BP monitors use arm cuffs but wrist cuff devices are also popular. Single cuff devices usually use the cuff pressure $(\mathrm{CP})$ data acquired from the cuff during cuff deflation. The pressure data from the cuff have the form of descending cuff pressure ramp which is modulated by arterial pulsations. The cuff arterial pulsations have relatively low amplitudes, typically 1$4 \mathrm{mmHg}$ and are usually separated from the CP ramp and amplified. The separation is accomplished by a high-pass filter. The CP ramp and the cuff pulse waveforms are shown in Figure 1. The arterial cuff pulses appear at the beginning of the gradual cuff deflation. Their amplitudes increase until they reach maximum and then they decrease until the end of the procedure.

Milan Stork's participation was supported by Department of Applied Electronics and Telecommunications, University of West Bohemia, Plzen, Czech Republic and by the European Regional Development Fund and the Ministry of Education, Youth and Sports of the Czech Republic under the Regional Innovation Centre for Electrical Engineering (RICE), project No. LO1607 and by the Internal Grant Agency of University of West Bohemia in Pilsen, the project SGS-2018-001
The cuff pulsations are frequently identified as oscillations and methods for automatic, single cuff BP determination are called oscillometric methods. Some single cuff methods for BP determination have been described in literature [1]. Manufacturers of BP devices consider algorithms for BP determination proprietary and keep them secret. It is necessary for efficient algorithm development to have a database of raw data from a BP cuff. Such proprietary databases exist and they are also kept secret. Some public databases of physiological signals exist $[2,3]$. Public databases of raw data from BP cuff do not exist at this time.

The secrecy surrounding commercial single cuff $\mathrm{BP}$ determination led us to the development of a public database of raw data from arm and wrist BP cuffs that can be downloaded by any interested investigator. The database can be used for development of BP algorithms and for algorithms for determination of some cardiovascular variables.

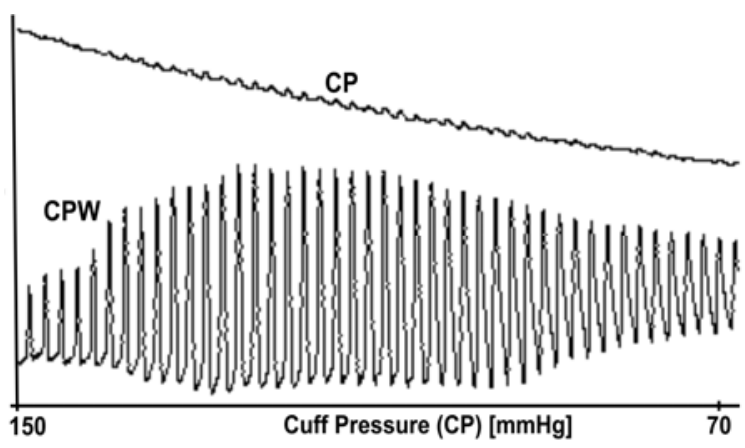

Figure 1. Cuff pressure (CP) ramp (upper trace) and amplified cuff pulse waveforms (CPW).

\section{METHODS}

a) Data acquisition electronics.

Previously developed dual cuff system [4] was used for acquisition, storage, and upload of raw data from two different cuffs. For simplicity we described only the parts of the system relevant to the above purpose. Block diagram in Figure 2 shows the relevant system components. The characteristics of these components are the results of careful observation and analysis of the raw data from pressure cuffs. 
Pressure in the cuff is converted to voltage in the piezoresistive pressure transducer and the output signal is amplified in the instrumentation amplifier. The low pass filter is a second order analog active filter with the cutoff frequency fc $=35 \mathrm{~Hz}$. The output of the low pass filter is connected to the CP input of the ADC and to the input of the high pass filter. The high pass filter is a second order analog active filter with the cutoff frequency fc $=0.5 \mathrm{~Hz}$. The $0.5 \mathrm{~Hz}$ cutoff frequency is sufficient for the removal of the $\mathrm{CP}$ descending ramp and for preservation of the low frequency components of cuff pulse waveforms. Higher cutoff frequencies can result in the $\mathrm{CP}$ waveform distortion, especially at low heart rates. At low heart rate and high cutoff frequency the diastolic segment of the waveform becomes depressed.

The output signal of the high pass filter is amplified and the output is connected to the CPW input of the 12-bit ADC with the conversion rate 85 samples/sec. The 2-channel digital data are converted into USB format and sent to a notebook computer. The data acquisition starts at the peak CP and ends at the end of the gradual cuff deflation. The acquired data are stored in binary files.



Figure 2. Block diagram of relevant cuff data acquisition electronics.

We used arm and wrist Omron commercial cuffs (Figure 3). The arm cuff bladder is $14 \mathrm{~cm}$ wide and 30 $\mathrm{cm}$ long. The wrist cuff bladder is $6 \mathrm{~cm}$ wide and 12 $\mathrm{cm}$ long. The data were acquired from sitting volunteers. The cuffs were kept at the level of the heart.

b) The database of raw data from BP cuffs.

We decided to present the database files as text files (file.txt). Text files can be easily converted from text to other digital formats for processing and for graphic display.

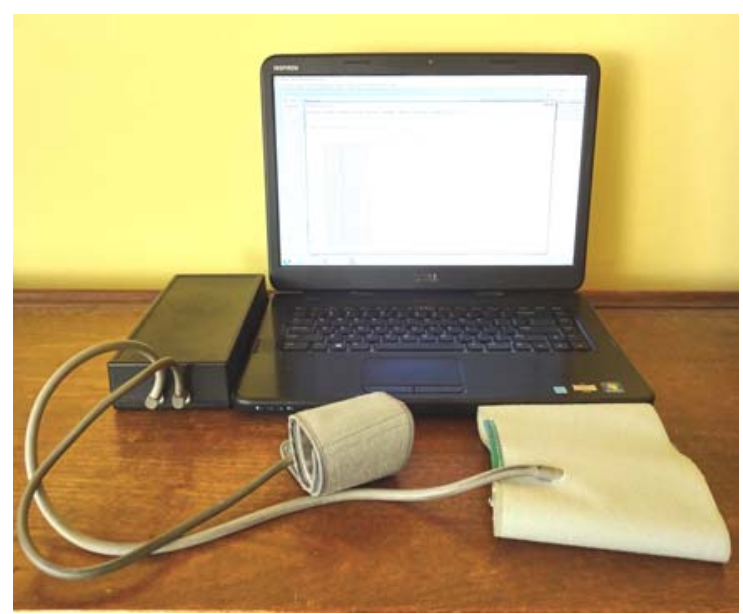

Figure 3. Block diagram of the wrist cuff sub-system consists of pneumatic and electronic circuits.

Each acquired cuff dataset is converted into a text file containing a series of two numbers. First number represents one point of cuff pressure and the second number is one point of the cuff pulse waveform. The time intervals between successive data pairs are 11.76 milliseconds. There are no other data contained in the files. Each file is identified by its name and number. Each name describes type of cuff (arm or wrist) and file number. For example, the name of first arm cuff file is ArmCuff(1).txt or WristCuff(1).txt. The files are stored in two folders. The arm cuff folder is named ArmCuff and the wrist cuff is named WristCuff.

Because the data files contain only cuff data, we created separate annotation documents. The documents list basic information about each file: File number - ArmCuff(n) or WristCuff(n), approximate systolic (SBP), mean (MAP), and diastolic (DBP) pressures, heart rate (HR), and heart rhythm. The approximate BP values are included in the annotation documents to provide the investigator an approximate idea what BP levels each data file represents. For example, SBP value of $150 \mathrm{mmHg}$ indicates hypertension and SBP value of $110 \mathrm{mmHg}$ indicates normal BP.

The method we used to compute the approximate SBP, MAP, and DBP values uses ratios of cuff pulse waveform amplitudes [5]. According to this method the SBP value corresponds to the point of $50 \%$ of maximal amplitude. Cuff pressure at the point of maximal CPW amplitude is MAP. The point of DBP is at the amplitude ratio of $75 \%$. The method is based on statistical estimates and it has inherent accuracy problems. Other single cuff BP methods have been described, but none of these methods or the commercial methods has been recognized as the "gold standard". The "gold standard" is still the manual method with a stethoscope and a sphygmomanometer [6]. Heart Rate (HR) value is computed from the intervals between CPW peaks. "Heart rhythm" 
describes irregularities of heart rhythm as seen during the duration of a test. There are three files containing atrial fibrillation (AF) data in the database

\section{RESULTS}

The database contains 30 arm cuff and 20 wrist cuff text files and two document files containing annotations for data files. The arm cuff and the wrist cuff data files are stored in two folders named ArmCuff and WristCuff. The annotation files are named ArmCuff.doc and WristCuff.doc. The database data and annotation files are uploaded to a Google Drive. Any interested party can download the database via link located at Carditech.org .

Each data text file contains series of two numbers. First number is the value of cuff pressure in $\mathrm{mmHg}$ and the second number reflects cuff pressure waveform $(\mathrm{CPW})$. The series start with the highest $\mathrm{CP}$ and the corresponding $\mathrm{CPW}$ and it ends with the lowest value of $\mathrm{CP}$ and the corresponding value of $\mathrm{CPW}$. The $\mathrm{CP}$ values are in $\mathrm{mmHg}$. The values of CPW are positive numbers corresponding to amplified cuff pulse values with positive offset. The annotation document (.doc) structure example is shown in the Table 1.

TABLE I. EXAMPLE OF THE STRUCTURE OF AN ANNOTATION DOCUMENT. BLOOD PRESSURE VALUES ARE IN MMHG, HEART RATE VALUES ARE IN BPM

\begin{tabular}{|c|c|c|c|c|}
\hline $\begin{array}{c}\text { File } \\
\text { number } \\
\text { (ArmCuff) }\end{array}$ & $\begin{array}{c}\text { SBP/DBP } \\
\text { (approx) }\end{array}$ & $\begin{array}{c}\text { MAP } \\
\text { (approx) }\end{array}$ & HR & $\begin{array}{c}\text { Heart } \\
\text { rhythm }\end{array}$ \\
\hline 1 & $121 / 80$ & 101 & 79 & normal \\
\hline 2 & $161 / 90$ & 127 & 50 & normal \\
\hline 3 & $111 / 78$ & 84 & 82 & normal \\
\hline
\end{tabular}

The range of approximate SBP values is 190-100 $\mathrm{mmHg}$ and the DBP range is $99-65 \mathrm{mmHg}$. The range of HR values is $46-94 \mathrm{bpm}$. Most of the files contain data with normal heart rhythm. One file contains data with irregular heart beats. Three arm cuff files have data acquired from volunteers with atrial fibrillation (AF) data.

\section{DISCUSSION}

Raw data from BP cuff have been used by investigators and manufacturers mainly for the development of algorithms for SBP, MAP and DBP values. Cuff pulse waveform contours have been mostly ignored by investigators. Our past research showed [7] that the waveforms have properties that make them suitable for the assessment of certain cardiovascular variables. We compared wrist cuff waveforms to radial artery waveforms obtained by direct method with catheter inserted into the radial artery and by applanation tonometry [8] which uses a pressure probe applied to the radial artery externally. The wrist cuff waveforms obtained at or below the point of DBP were similar to the waveforms obtained by the direct and the tonometry methods. The arm cuff waveforms are less suitable for waveform analysis because the arm cuff contains substantial amount of air and many arms have large amount of soft tissue. The air and the tissue contribute to dampening of the waveforms. This effect can be seen in Figure 4. The bottom arm cuff waveforms show rounding of the peaks of waveforms while the wrist cuff waveforms do not show pronounced rounding.

Application of wrist cuff pulses for BP determination is controversial. Typical wrist cuff width in commercial devices is about $6 \mathrm{~cm}$, which is too narrow for many individuals. Cuff width should be $40 \%$ of wrist circumference [9]. For example, cuff width for $20 \mathrm{~cm}$ wrist circumference is $8 \mathrm{~cm}$. Our previous study [10] showed the effect of under-cuffing on accuracy and reliability of wrist cuff BP devices. Investigators interested in wrist cuff BP determination should take this into account. We want to emphasize that the wrist cuff data is more suitable for investigating the waveform contour properties than for BP determination.

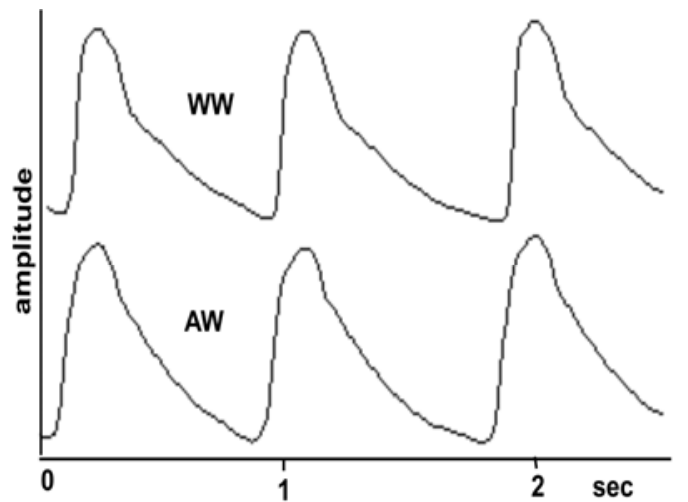

Figure 4. Cuff pulse waveforms acquired simultaneously from wrist (WW) and arm (AW) cuffs.

The arm cuff raw data from three tests of atrial fibrillation (AF) serve as examples of irregular heart rhythm. Figure 5 shows the irregularity in the timing and in the amplitude of the pulses. The resulting BP values are frequently inconsistent.

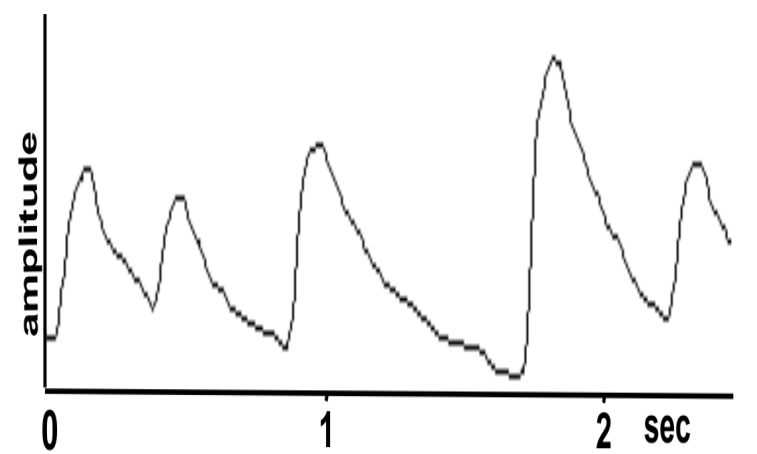

Figure 5. Cuff pulse waveforms acquired from a volunteer with atrial fibrillation.

\section{CONCLUSION}

The described public database of raw data from two different BP cuffs provides free data to interested investigators. The database does not provide calibrated, accurate BP values. To obtain calibrated values a validation protocol [11] would have to be followed. Our aim was to provide an introductory data 
that still contain valuable information. The database can be expanded in the future.

\section{REFERENCES}

[1] J Jilek and T Fukushima, "Oscillometric Blood Pressure Measurement: The Methodology, Some Observations, and Suggestions", Biomed Instrum \& Technol 2005, vol. 19, pp 237-241.

[2] A L Goldberg, L A Amaral, L Glass, J M Hausdorff, P C Ivanov and R G Mark, "Physiobank, PhysioToolKit, and PhysioNet: Components of a New Research Resource for Complex Physiologic Signals", Circulation 2000, vol 101, pp 215-220.

[3] B E Westerhof, J Gisof, W J Stok, K H Wesseling and J M Karemaker, "Time-domain crosscorrelation baroreflex sensitivity; performance on the EUROBAVAR data set", J Hypertens 2004, vol 22, pp 1-10.

[4] J Jilek and M Stork, "Dual Cuff System Improves Noninvasive Blood Pressure Determination" , Proc. of Int. Conf. Applied Electronics, Plzen, Czech Republic 2010.

[5] L A Geddes, "Characterization of the oscillometric method for measuring indirect blood pressure," Ann. Biomed. Eng. 1982, vol. 10, pp. 271-280.
[6] D W Jones, L J Appel, S D Sheps, E J Rocella and C Lenfant, "Measuring Blood Pressure Accurately", JAMA 2003, vol 289, pp 1027-1030.

[7] M Stork and J Jilek, "Cuff Pressure Pulse Waveforms: Their Current and Prospective Application in Biomedical Instrumentation" in: A $\mathrm{N}$ Laskovski ed. Biomedical Engineering, Trends in Electronics, Communication and Software, Intech 2011, pp 193-210.

[8] J Doupis, N Papanas, A Cohen, L McFarlan and E Horton, "Pulse wave Analysis by Applanation Tonometry for the Measurement of Arterial Stiffness", The Open Cardiovascular Medicine Journal 2016, vol 10, pp 188-195.

[9] T G Pickering, J E Hall, L J Appel, B E Falkner, J Graves, M N Hill, D W Jones, T Kurtz, S G Sheps and E J Rocella, "Recommendations for blood pressure measurement in humans and experimental animals: part 1, Blood pressure measurement in humans", Hypertension 2005, vol 45, pp 142161.

[10] J Jilek, M Stork, "Cuff width alters the amplitude envelope of wrist cuff pressure pulse waveforms", Physiological Measurement 2010, vol 10, pp 43-49.

[11] E O'Brien, N Atkins, G Stergiou, N Karpettas, G Parati, R Asmar, Y Imai, J Wang, T Mendgen and A Shennan, "European Society of Hypertension International Protocol revision 2010 for the validation of blood pressure measuring devices in adults", Blood Pressure Monitoring 2010, vol 1, pp 23-38. 